\title{
Origin of Orbital Ferromagnetism and Giant Magnetic Anisotropy at the Nanoscale
}

\author{
A. Hernando, P. Crespo, and M. A. García \\ Instituto de Magnetismo Aplicado, RENFE-UCM-CSIC, and Departamento de Física de Materiales UCM, P.O. Box 155, \\ Las Rozas, Madrid 28230, Spain
}

(Received 31 October 2005; published 7 February 2006)

\begin{abstract}
The origin of orbital magnetism recently observed in different nanostructured films and particles is discussed as a consequence of spin-orbit coupling. It is shown that contact potentials induced at the thin film surface by broken symmetries, as domain boundaries in self-assembled monolayers, lead to orbital states that in some cases are of large radius. The component of the angular momentum normal to the surface can reach very high values that decrease the total energy by decreasing spin-orbit interaction energy. Intraorbital ferromagnetic spin correlations induce orbital momenta alignment. The estimated values of the magnetic moments per atom are in good agreement with the experimental observations in thiol capped gold films and nanoparticles.
\end{abstract}

DOI: 10.1103/PhysRevLett.96.057206

PACS numbers: 75.10. $-\mathrm{b}, 73.20 .-\mathrm{r}, 75.75 .+\mathrm{a}$

A set of amazing independent experimental results on the magnetic properties of some particular nanostructures has been recently published. Ferromagnetic hysteresis in proton irradiated graphite with the Curie temperature ranging between 500 and $800 \mathrm{~K}$ was observed and reported [14]. Permanent giant magnetic moments were detected at the surface layers of thin films of oxides and borides [5-7]. Ferromagnetic hysteresis at room temperature was measured in $1.7 \mathrm{~nm}$ gold nanoparticles (NPs) surrounded by thiols [8]. Highly anisotropic giant moments were also observed for self-organized organic molecules linked by thiols bonds to gold films [9]. A first remarkable characteristic common to all these observations is that the well known theory of magnetism based upon the unfilled character of $3 d$ or $4 f$ electron energy levels cannot be directly applied to these cases. A second property is that the local anisotropy seems to be extremely high as compared to the typical anisotropy strength of the well known harder materials. The third important feature deals with the thermal dependence of magnetization. For both types of observed magnetism, giant paramagnetism and ferromagnetism alike, the thermal dependence of magnetization does not obey the usual Curie, Curie-Weiss, or Bloch law as expected, respectively, from the classical theory of magnetism. In contrast, the magnetization seems to be almost independent of temperature between 0 and $300 \mathrm{~K}$.

In this Letter, we will focus on magnetism of thiol capped gold films and NPs. It is important to note that, for the case of gold capped with thiol groups, there exists a great difference between the magnetic behavior observed for NPs and for thin films. For both cases, a large anisotropy can be detected, by direct measurements in thin films [9] and by blocking temperature considerations in NPs [8]. However, whereas the magnetic moment reaches giant values (10 or even $100 \mu_{B}$ per atom) for films, it is extremely low $\left(0.01 \mu_{B}\right.$ per atom) for NPs. There is experimental evidence associating the appearance of magnetic properties with the charge transfer that takes place by thiol bonding. Electron transfer of about $0.1 e$ per atom from the gold $5 d$ energy level to the organic molecules has been observed by $\mathrm{x}$-ray-absorption near-edge spectroscopy $[8,10]$. Uncapped gold thin films as well as uncapped gold NPs have been shown to be diamagnetic as bulk gold.

It is well known that the organic molecules form closepacked self-assembled monolayers (SAMs) and exhibit cooperative electronic properties [11]. SAMs spontaneously form domains [12]. For incomplete monolayers, patches of the monolayer coexist with patches of the bare metal. The domains observed so far have sizes ranging from nanometers to a micrometer. According to electron circular dichroism measurements, carried out on thiolated organic monolayers on gold [13], the orbital momentum has a uniform orientation within each domain. Therefore, the origin of the observed magnetic moment should be associated with this orbital momentum. The presence of long range orbital ferromagnetism also can account for the giant atomic magnetic moments experimentally observed. Orbital momenta can increase enormously, as has been reported for the cases of giant diamagnetism [14], when the orbital radius is large enough to smooth the increasing rate of kinetic energy. Orbital magnetism also is associated with giant magnetic anisotropy, since rotation of the angular momenta requires the rotation of the electric charge distribution fixed in orientation by electrostatic interactions.

In order to reach a deeper understanding of the phenomenon, the following two aspects should be explained: the ferromagneticlike coupling between orbital momenta and the origin of the giant orbital momentum per atom. An interesting attempt to account for the giant magnetic moments, in gold films capped with SAMs, has been carried out by Vager and Naaman [15]. They considered electrons pumped up from the substrate to the molecular layer coupled in pairs forming bosonlike triplets of spin 1 . Based on these statements. they used the following $L_{z}$ dependent Hamiltonian, where $z$ holds for the direction 
normal to the film plane:

$$
H_{o}=\frac{L_{z}^{2} \hbar^{2}}{2 m \xi^{2}}-\frac{q \hbar}{2 m} L_{z} B .
$$

It is immediately observed that minimization of the energy with respect to $L_{z}$ yields a value proportional to $B$ and to the square of the orbital radius $\xi$. However, as we have previously reported [16], the Hamiltonian considered by Vager and Naaman missed the diamagnetic term, that when included leads to a vanishing total magnetic moment in perfect agreement with Van Leeuwen theorem [17].

It is shown here that the form of the Hamiltonian (1) can be recovered by considering spin-orbit effects that are known to be extremely important in gold surfaces and do not include diamagnetic effects.

Spin-orbit splitting has been experimentally observed by LaShell et al. [18] for the $s p$-derived surface state on $\mathrm{Au}(111)$. This splitting is a consequence of the lack of inversion symmetry that is broken at the surface. The free electron model leads to splitting of $10^{-6} \mathrm{eV}$, several orders of magnitude smaller than $0.41 \mathrm{eV}$ that is the measured value. However, Petersen and Hedegard [19], using a tightbinding model, have shown that the order of the splitting strength for the $p$ band $\alpha_{r}$ can be explained by considering its dependence on the spin-orbit interaction of the atomic levels as well as on the surface potential. They found the following relationship: $\alpha_{r}=6 \alpha \gamma / \omega$, where $\alpha$ is the atomic spin-orbit parameter. The parameter $\gamma$ corresponds to the overlap matrix element of any pair of $p$ states with lobes parallel to the surface and that with a perpendicular one, and $\omega$ is the band width. The term $\gamma$ reflects the direction of broken symmetry and is a measure of the potential gradient.

For a free gold surface, the electric field is perpendicular to the surface and the spin-orbit coupling permits surface state levels with the same parallel wave vector $k$ and opposite spins to have different energies. However, when a region of the surface is capped with chemisorbed organic molecules, a potential gradient (contact potential) extended along the screening length appears at the boundary of the region. This effect is induced by charge transfer associated with binding. The contact potential can be measured by Kelvin microscopy [13,20]. Ichii et al. [21] have measured surface potential by noncontact atomic force microscopy in capped gold films and have found that the voltage increases linearly with the chain length at a rate of $9 \mathrm{mV}$ per $\mathrm{CH}_{2}$ unit. Therefore, the difference of potential $U$ values between capped and uncapped regions of the surface is normally comprised between 50 and $100 \mathrm{mV}$. Let the capped region be a circular domain of radius $\xi$. As a consequence of the contact potential $U$, a radial electric field $E=-(d U / d r)_{r=\xi}$ is induced at the domain boundary. Quasifree electrons of the Au surface can be eventually captured in atomiclike orbitals of large radius $\xi$ at the domain boundary potential step. If the spin component of the electrons along the $z$ axis is $s_{z}$, the $L_{z}$ dependent part of the Hamiltonian $H$ can be written as

$$
H=\frac{L_{z}^{2} \hbar^{2}}{2 m \xi^{2}}-\alpha_{r} L_{z} s_{z} \hbar^{2} .
$$

The $L_{z}$ value that minimizes (2) is given by

$$
L_{z}=m \xi^{2} \alpha_{r} s_{z} .
$$

From (3), it is obvious to observe that the $L_{z}$ dependent orbital energy is invariant under simultaneous inversion of $L_{z}$ and $s_{z}$, i.e., $\varepsilon\left(L_{z}, s \uparrow\right)=\varepsilon\left(-L_{z}, s \downarrow\right)$. The minimum orbital energy, corresponding to quantum number $L_{z}$ given by (3), is $\varepsilon_{\min }=-(1 / 2) m \xi^{2} \alpha_{r}^{2} s_{z}^{2} \hbar^{2}$. This energy term is negative between $L_{z}=0$ and $\left|L_{z}\right|=2 m \xi^{2} \alpha_{r} s_{z}$ and vanishes at both interval limits. Since the magnitude of the spin-orbit splitting can be understood only from atomic fields ( $\gamma$ values), not only from free electrons in the electric field produced by the surface potential step, it is reasonable to assume a value for $\alpha_{r}$ of the same order than that measured for free gold surfaces, i.e., $\alpha_{r} \hbar^{2}=0.4 \mathrm{eV}$. Figures 1(a)-1(c) illustrate, for a single electron approximation, the orbital energy $\varepsilon$, dependence on the quantum number $L_{z}$, for $\alpha_{r} \hbar^{2}=0.4 \mathrm{eV}$, and three values of $\xi$, respectively. As observed in Fig. 1, the orbital momentum can reach giant values for values of $\xi$ as those experimentally observed that are comprised between 10 and $10^{3} \mathrm{~nm}$.

Now the number of electrons that can be accommodated in the localized atomiclike states at the orbit with radius $\xi$ should be estimated. This number corresponds to the number of orbital states with energy below the Fermi energy $E_{F}=5.5 \mathrm{eV}$ of the quasifree electrons of the gold surface. The band population is more relevant as $E_{F}-\varepsilon_{\min }$ increases, i.e., for large $\xi \alpha_{r} \hbar$. The number of electrons with spin up that can be trapped by decreasing the energy is at least the number of possible states comprised between $L_{z}=0$ and $L_{z}=2 m \xi^{2} \alpha_{r} s_{z}$. Note that we have neglected those localized states with orbital energy comprised between 0 and $E_{F}$ that is correct when $\varepsilon_{\min } \ll E_{F}$ [22]. The values of $\varepsilon_{\text {min }}$ plotted in Fig. 1 also directly indicate that the condition is accomplished for the assumed $\xi$ and $\alpha_{r} \hbar$ values. The maximum $L_{z}$ is given by the integer closer to $m \xi^{2} \alpha_{r}$. Since the average $L_{z}$ is $m \xi^{2} \alpha_{r} s_{z}$, the total orbital momentum associated with the electrons of spin up trapped in the orbit becomes $m^{2} \xi^{4} \alpha_{r}^{2} / 2$. The total orbital momentum per surface atom included within the domain is of the order of $m^{2} \xi^{2} \alpha_{r}^{2} a^{2}$, where $a$ is an effective lattice constant. The same magnitude of angular momentum but with opposite direction should correspond to the electrons with spin down trapped in the orbit. However, electron circular dichroism observations [13] indicate that each domain is characterized by a well defined angular momentum. Thereby, it can be inferred that there is spin unbalance between the electrons occupying the orbit and, consequently, a permanent magnetic moment with contributions coming from both spin and giant orbital momenta. Such 

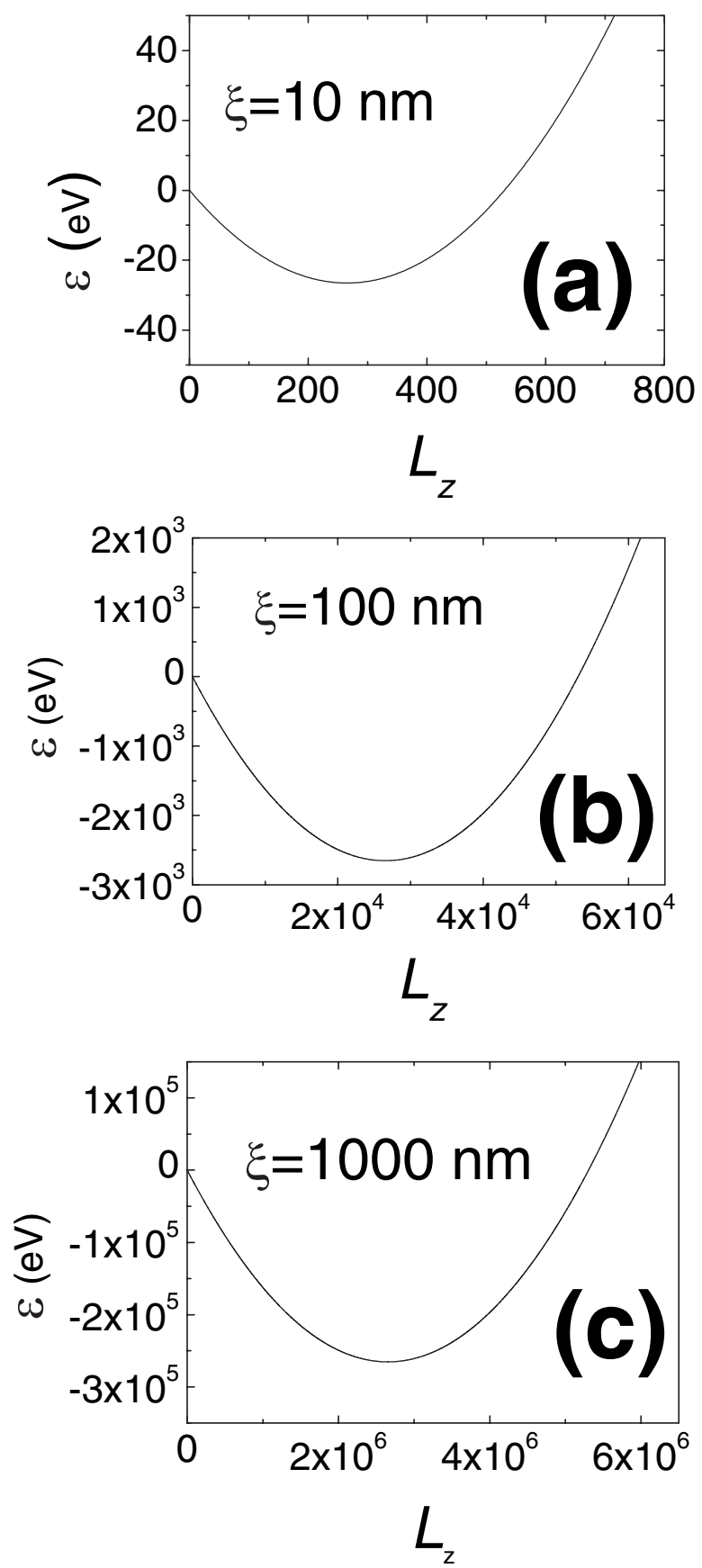

FIG. 1. (a)-(c) Orbital energy $\varepsilon$ of the electron in the localized orbit as a function of $L_{z}$ for different radius.

ferromagnetic arrangement of the orbital momenta can be explained as a consequence of the ferromagnetic spin configuration, as outlined below.

Electrostatic interactions between the huge number of electrons concentrated in the orbit, and separated between them an average distance $2 \pi / m \xi \alpha_{r}^{\prime}(0.01 \AA$ for $\xi=$ $10^{-6} \mathrm{~m}$ ), tend to decrease through spin alignment, similarly to the effect described by the Hund rule in atomic orbitals. As calculated by Vager and Naaman [15] for these distances between a pair of electrons, the electrostatic energy difference between the singlet and triplet configuration is of many $\mathrm{eV}$. Even though the total occupation of both spin up and spin down subbands would produce further decrease of the orbital energy, the rapid increase of electrostatic repulsive energy counterbalances these two trends and defines a spin unbalanced final occupation of the band. As the orientation of $L_{z}$ is linked to that of $s_{z}$ through the spin-orbit coupling, spin alignment implies orbital momenta alignment.

If we assume all the trapped electrons to be spin polarized (full occupation of only the spin up subband), the number of Bohr magnetons per surface atom linked to an organic molecule and associated with the orbital angular momentum is given by $n\left(\mu_{B}\right)=m^{2} \xi^{2} \alpha_{r}^{2} a^{2}$. It is worth noting that $n\left(\mu_{B}\right)$ can reach very high values even though we have taken into account the fermionic character of the electrons. Figure 2 illustrates $n\left(\mu_{B}\right)$ as a function of $\xi$ for different values of $\alpha_{r}$ as well as a comparison with experimental data. The $n\left(\mu_{B}\right)$ expression accounts for the more relevant experimental aspects observed in the magnetism of organic molecule capped gold nanoparticles. (i) The magnetic moment comes mainly from the orbital momentum that implies high magnetic anisotropy. (ii) Quasifree electrons are trapped in localized states giving rise to a drastic decrease of the electron mobility as observed by surface plasmon resonance measurements [8]. (iii) The magnetic moment being of orbital origin can reach giant values if $\xi$ is sufficiently large, as is the case for selfassembly domains in thin films. (iv) The origin of orbital moment alignment is the exchange interactions between spins. (v) Degradation of the organic layer leads to a

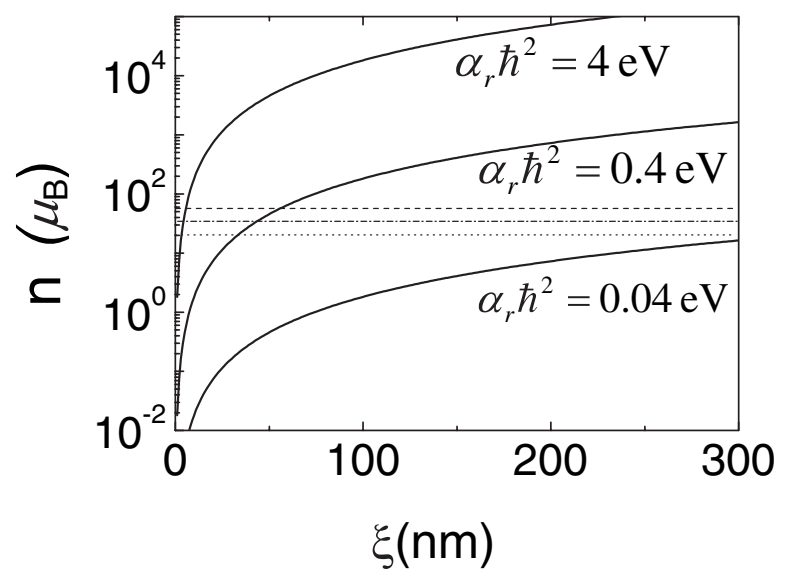

FIG. 2. Magnetic moment, in number of Bohr magnetons, per surface atom [Au (111)] as a function of the orbit radius for different values of $\alpha_{r}$. Experimental results reported and measured [9] are also plotted as horizontal lines: (dashed line) fresh samples; (dashed-dotted line) aged 5 days; (dotted line) aged 10 days. The effect of aging is a decrease of the domain size as observed in Ref. [12]. If we consider $\alpha_{r} \hbar^{2}=0.4 \mathrm{eV}$, the average domain radius decreases from $70 \mathrm{~nm}$ in fresh samples to 45 and $30 \mathrm{~nm}$ after for samples aged for 5 and 10 days, respectively. 
modification of $\xi$ and a consequent degradation of the magnetic properties $[9,12]$ (see also Fig. 2). (vi) By increasing the chain length of the capping molecule, an increase in both potential [21] and in magnetic moment [9] have been experimentally observed. Both effects are related to the increase of charge transfer that reinforces the spin-orbit interaction strength $\alpha_{r}$ and, consequently, the orbital momentum according to (3). (vii) Since the $z$ axis should be well defined through the domain-plane surface - the value of $\xi$ is necessarily very small for the case of nanoparticles where the $z$ direction changes in an interatomic distance ( $\xi$ is in this case of the order of the lattice constant). Consequently, the magnetic moment per atom is more than 3 orders of magnitude smaller than that observed for some thin films [8], as illustrated by Fig. 1. Note that, by increasing the particle size, the decreasing rate of the total fraction of surface atoms makes the magnetic contribution of the surface to be masked by the core diamagnetism. As the atomic moment is so small, it can be detected only for a very small size, when the diamagnetic contribution of the volume is negligible. This is the reason why ferromagnetism is observed only for very small particles.

As has been shown, the combination of spin-orbit coupling $\alpha_{r}$ and contact potential at the large radius $\xi$ domain boundaries can account for the existence of a giant orbital moment induced in atomiclike localized states. The contact potential is originated by the electron holes induced by electron transfer to the organic molecule. Electrostatic interaction between electrons trapped in the orbit yields spin alignment that, through spin-orbit coupling, induces subsequent orbital moment alignment. The basis of the argument used here to account for the high population of the orbital band, large $\xi \alpha_{r} \hbar$, could be extended to other sources of broken symmetry as, for instance, twin boundaries [23] or groups of defects induced by irradiation [1-4]. In summary, a new type of magnetic order has been described.

A. H. is indebted to Dr. J. M. Rojo and Dr. F. Briones for critical and helpful discussions.

[1] S. Talapatra et al., Phys. Rev. Lett. 95, 097201 (2005).

[2] A. V. Rode et al., Phys. Rev. B 70, 054407 (2004).
[3] J. M.D. Coey, M. Venkatesan, C. B. Fitzgerald, A.P. Douvalis, and I. S. Sanders, Nature (London) 420, 156 (2002).

[4] T. L. Makarova, Semiconductors 38, 615 (2004).

[5] K. Kusakabe and M. Maruyama, Phys. Rev. B 67, 092406 (2003); P. O. Lehtinen, A. S. Foster, Y. Ma, A. V. Krasheninnikov, and R. M. Nieminen, Phys. Rev. Lett. 93, 187202 (2004).

[6] L. S. Dorneles, M. Venkatesan, M. Moliner, G. Lunney, and J. M. D. Coey, Appl. Phys. Lett. 85, 6377 (2004).

[7] M. Venkatesan, C.B. Fitzgerald, and J. M.D. Coey, Nature (London) 430, 630 (2004).

[8] P. Crespo et al., Phys. Rev. Lett. 93, 087204 (2004).

[9] I. Carmelli, G. Leitus, R. Naaman, S. Reich, and Z. Vager, J. Chem. Phys. 118, 10372 (2003).

[10] P. Zhang and T. K. Sham, Phys. Rev. Lett. 90, 245502 (2003).

[11] A. Ulman, Chem. Rev. 96, 1533 (1996).

[12] E. Barrena, C. Ocal, and M. Salmerón, J. Chem. Phys. 111, 9797 (1999); K. Tamada, M. Hara, H. Sasabe, and W. Knoll, Langmuir 13, 1558 (1997); Z. Suo, Y. F. Gao, and G. Scoles, J. Appl. Mech. 71, 24 (2004).

[13] Z. Vager, I. Carmeli, G. Leitus, S. Reich, and R. Naaman, J. Phys. Chem. Solids 65, 713 (2004); see also spin polarized results reported in Y. Yamamoto et al., Phys. Rev. Lett. 93, 116801 (2004).

[14] A. Hernando et al., Phys. Rev. B 56, 7800 (1997).

[15] Z. Vager and R. Naaman, Phys. Rev. Lett. 92, 087205 (2004).

[16] A. Hernando and M. A. Garcia, Phys. Rev. Lett. 96, 029703 (2006).

[17] J. A. Van Leeuwen, J. Phys. Radium 6, 361 (1921).

[18] S. LaShell, B. A. McDougall, and E. Jensen, Phys. Rev. Lett. 77, 3419 (1996); see also Y. M. Koroteev et al., Phys. Rev. Lett. 93, 046403 (2004).

[19] L. Petersen and P. Hedegard, Surf. Sci. 459, 49 (2000).

[20] T. Ichii, T. Fukuma, K. Kobayashi, H. Yamada, and K. Matsushige, Appl. Surf. Sci. 210, 99 (2003).

[21] T. Ichii, T. Fukuma, H. Yamada, and K. Matsushige, Nanotechnology 15, S30 (2004).

[22] $\left|\varepsilon_{\text {min }}\right|$ is assumed to be also larger than the electrostatic energy eU (approximately lower than $0.1 \mathrm{eV}$ ) acting on the captured electrons. Similar estimations of band population and average $L_{z}$ can be easily performed for different relative positions of $E_{F}$ with respect to $\varepsilon_{\min }$, provided that $E_{F}>\varepsilon_{\text {min }}+$ eU. If $E_{F}<\varepsilon_{\text {min }}+\mathrm{eU}$, the orbital band should be empty at low temperatures.

[23] B. Sampedro et al., Phys. Rev. Lett. 91, 237203 (2003). 